Laura Ascone, University of Lorraine, France

DOI: 10.17951/Ismll.2020.44.1.125-134

\title{
La spontanéité des émotions mise à l'épreuve sur internet : exprimer, susciter, manipuler
}

\author{
The Spontaneity of Emotions Challenged by the Internet: \\ Expressing, Eliciting, Manipulating
}

\section{RÉSUMÉ}

Face au développement de la communication médiée par les réseaux, l'expression des émotions a subi d'importants changements. Cette contribution propose une analyse linguistique de l'expression des émotions sur internet. Afin d'examiner la façon dont l'expression des émotions peut être exploitée par l'énonciateur, l'étude a porté sur un discours propagandiste diffusé sur internet. Plus particulièrement, Dabiq et Dar al-Islam, les deux revues publiées en ligne par l'État islamique, ont constitué notre corpus. Il a été ainsi possible de voir comment les émotions, par nature spontanées, se traduisent ici en productions soigneusement formulées.

Mots-clés : émotions, expression, internet, propagande

\section{ABSTRACT}

Due to the development of the computer-mediated communication, the expression of emotions has undergone significant changes. This paper proposes à linguistic analysis of the verbalisation of emotions on the Internet. The goal was to examine the way the expression of emotions can be exploited by the speaker. Therefore, the study was conducted on a propagandist discourse available on the Internet. More particularly, Dabiq and Dar alIslam, the official online magazines published by the Islamic State, constituted the corpus of this analysis. This way, it was possible to investigate how emotions, which are spontaneous by nature, become carefully formulated productions.

Keywords: emotions, expression, Internet, propaganda

\section{Introduction}

Sujet d'étude depuis l'antiquité, l'émotion a suscité l'intérêt de plusieurs disciplines, parmi lesquelles figurent la philosophie, la psychologie, la neurologie et la linguistique. Cependant, malgré les nombreuses analyses menées dans les différents domaines, l'étude des émotions est loin d'être exhaustive. Cette contribution propose une analyse linguistique de l'expression des émotions dans un discours

\footnotetext{
Laura Ascone, UFR Sciences Humaines et Sociales - Laboratoire CREM, Université de Lorraine, lle du Saulcy BP 30309, 57006 Metz Cedex 1, laura.ascone@univ-lorraine.fr, https://orcid.org/0000-0002-7595-1156
} 
propagandiste diffusé sur internet. Cette approche nous a permis d'examiner la façon dont la communication médiée par les réseaux influence l'expression de nos réactions émotionnelles. De même, elle a montré comment le discours propagandiste recourt à l'expression et à l'élicitation des émotions afin de moduler le comportement de son destinataire.

L'étude a été menée sur le discours propagandiste produit par l'État islamique, que nous appellerons ici Daesh, et diffusé sur internet. Plus particulièrement, le corpus a été constitué à partir des articles publiés dans les quinze numéros de Dabiq et les dix numéros de Dar al-Islam, les revues publiées en ligne par Daesh depuis juillet 2014. L'adoption d'une approche qualitative ${ }^{1}$ nous a permis d'examiner les spécificités de l'expression des émotions dans le discours djihadiste.

Après avoir examiné la façon dont les émotions sont exprimées dans le cyberespace, nous présenterons comment Daesh recourt aux émotions dans son discours à travers une mise en scène et une manipulation des émotions. Enfin, nous verrons comment deux émotions opposées telles que la peur et la joie s'articulent au sein d'un même discours.

\section{L'expression des émotions sur internet}

Le cyberespace est généralement considéré comme l'une des causes de la déshumanisation des relations interpersonnelles. Cependant, il a rendu possible le développement de nouvelles formes d'expression des émotions. Réseaux sociaux, forums et sites web permettent à l'internaute d'exprimer ses propres opinions et ses propres émotions. Par nature spontanées, les réactions émotionnelles se traduisent ici en productions soigneusement formulées.

\subsection{Une approche théorique de l'analyse linguistique des émotions}

La notion et l'étude des émotions sont présentes depuis l'antiquité. Si le Timée de Platon présente les passions comme des forces qui nous font perdre l'équilibre, selon Sartre (1962) les passions nous permettent de comprendre notre existence dans le monde. De même, si pendant le Siècle des Lumières les émotions étaient perçues comme quelque chose d'irrationnel qui devait être réprimé, le Romantisme a placé les émotions au cœur d'œuvres littéraires et d'études scientifiques. Parmi les disciplines qui étudient ce phénomène, nous retrouvons la psychologie, qui s'intéresse au lien entre les émotions et la cognition (Christophe, 1998; Scherer, 2001; Marsella \& Gratch, 2009), la pragmatique, qui analyse la relation entre les émotions et l'action (Caffi \& Janney, 1994), et la linguistique, qui vise à examiner la façon dont les émotions sont exprimées verbalement.

\footnotetext{
1 Cette analyse qualitative a été menée à partir des résultats issus d'une analyse quantitative réalisée avec les logiciels Tropes et Iramuteq (Ascone, 2018).
} 
En ce qui concerne l'analyse linguistique, il est important de rappeler que selon Sapir (1921) l'expression des émotions n'est pas de nature linguistique (pp. 38-40). Cependant, Harkins et Wierzbicka (2001) et Scherer (2005) affirment que le langage constitue le seul moyen nous donnant la possibilité de mesurer l'expérience émotionnelle. Plus particulièrement, le langage nous permet d'accéder à la façon dont l'individu interprète et catégorise l'épisode émotionnel qu'il a vécu (p. 712)2. C'est principalement pour cette raison que la linguistique a examiné l'expression des émotions de plusieurs points de vue : syntaxique, sémantique ${ }^{3}$, psycholinguistique, etc. L'expression verbale des émotions permet à l'énonciateur de matérialiser ce qu'il a ressenti, et à son interlocuteur de comprendre le concept abstrait auquel l'énonciateur fait référence. Une attention particulière a été portée sur la distinction entre le discours émotionnel et le discours émotif (Plantin, 2011). Plus particulièrement, dans le discours émotionnel, l'émotion est exprimée à l'instant même où elle est ressentie. Au contraire, dans le discours émotif le locuteur décrit ce qu'il a ressenti une fois que l'émotion s'est dissipée. Autrement dit, le discours émotif se rapproche davantage de la rhétorique et de la persuasion (Arndt \& Janney, 1991). Le pathos joue ici un rôle crucial : en faisant appel aux émotions du destinataire, l'orateur base son argumentation sur l'empathie avec celui-ci. Dans la section suivante, nous verrons comment le cyberespace influence aussi bien l'expression que la description de ces réactions émotionnelles.

\subsection{S'exprimer sur internet}

La création d'internet et son développement dans les systèmes de communication ont changé la façon dont nous nous rapportons à notre environnement et à notre entourage. Dans la communication médiée par les réseaux, ce qui a subi un changement important ce sont les notions de temps et d'espace. En ce qui concerne l'axe du temps, l'internaute perçoit les interactions virtuelles instantanées et temporellement fluides, exactement comme les interactions réelles. Toutefois, ces interactions sont marquées par plusieurs interruptions : chaque fois qu'il envoie un message, l'utilisateur doit attendre que son interlocuteur lise le message, rédige et envoie une réponse. Quant à l'axe de l'espace, bien que l'internaute soit devant l'ordinateur dans le monde réel et que les messages apparaissent sur l'écran, les interlocuteurs ne se trouvent pas dans le même espace (Kramsch, 2009).

Grâce à ce décalage spatio-temporel, propre aux interactions médiées par les réseaux, l'énonciateur peut aussi bien se présenter à travers une autre identité que

2 Language "is the only available means to measure the subjective experience of an emotion, as it is our only access to the way an individual interprets and categorizes an emotion episode s/he experiences" (Scherer, 2005, p. 712).

3 Parmi ces études, on retrouve les travaux de Goossens (2005) qui a élaboré une typologie des noms de sentiment qui reflète leurs propriétés combinatoires et qui décrit leurs caractéristiques sémantiques. 
cacher ses intentions derrière des énoncés plus ou moins véridiques. Par conséquent, ce décalage a un impact non négligeable sur les interactions ainsi que sur la manière dont les interlocuteurs verbalisent leurs émotions. Lorsque l'énonciateur veut exprimer une émotion sur internet, il tiendra compte du contexte de production et, plus particulièrement, du moyen de communication utilisé, du type de conversation et de son interlocuteur. Le contexte dans lequel l'internaute s'exprime influence donc son discours. De même, ce que l'énonciateur dit, et plus spécifiquement la façon dont il le dit, module les réactions de son interlocuteur ainsi que la conversation même. Autrement dit, en décidant comment exprimer une émotion, l'énonciateur décide comment agir sur son interlocuteur. Plus particulièrement, l'internaute agit sur son interlocuteur car l'interprétation de ce dernier dépend principalement de la manière dont l'émotion a été verbalisée. Cela est rendu possible par le fait que, comme il a déjà été affirmé, le langage est le seul moyen d'interpréter toute réaction émotionnelle (Scherer, 2005). Kramer, Guillory, et Hancock (2014) ont étudié l'impact qu'une émotion exprimée sur Facebook a sur les réactions émotionnelles des autres internautes. Les utilisateurs exposés à un nombre important de messages négatifs ont tendance à publier des contenus négatifs.

Comme nous l'avons montré, les émotions et la façon dont elles sont exprimées occupent une place centrale aussi bien dans les interactions réelles que dans les interactions virtuelles. Lorsque l'énonciateur communique sur internet, ses émotions se dissipent instantanément, avant qu'il n'ait le temps de les exprimer par écrit. Cela dépend du fait que les émotions durent seulement quelques secondes (Ekman, 1992). L'internaute pourra donc décider, plus ou moins volontairement, comment formuler l'expression de ses émotions. Internet met ainsi à l'épreuve la spontanéité de ces réactions émotionnelles. Par conséquent, la communication virtuelle peut être considérée comme une communication émotive et non pas émotionnelle. Sur internet, l'énonciateur peut facilement mettre en scène ses émotions afin de moduler les réactions et le comportement de son interlocuteur.

\section{L'exploitation des émotions dans la propagande djihadiste}

Le discours de propagande djihadiste diffusé sur internet constitue un exemple évident de l'exploitation des réactions émotionnelles. Dans cette section, nous examinerons la manière dont Daesh utilise l'expression des émotions afin d'amener l'individu à adhérer à l'idéologie djihadiste ou, dans certains cas, à agir au nom de cette idéologie.

\subsection{Exprimer et mettre en scène une émotion pour inciter à agir}

Le terme terrorisme dérive du mot latin terror, dont la racine indoeuropéenne ter- signifie « trembler ». Ce lien étymologique entre le terrorisme et la peur (Di Cesare, 2017) se traduit dans toute action terroriste, dont l'objectif est de semer la peur. De même, le discours de propagande terroriste témoigne de la contiguité 
entre la peur et l'action terroriste. Comme nous le verrons dans cette deuxième partie, le discours djihadiste montre clairement l'importance d'exprimer et de susciter la peur. Lors de la proclamation de la réinstauration du califat, le 4 juillet 2014 à Mossoul, le leader de Daesh Abu Bakr Al-Baghdadi a affirmé :

(1)

Revenir à l'Islam des premiers âges pour obtenir le pardon d'Allah et retrouver la fierté arabe en inspirant la peur aux infidèles et aux mauvais musulmans.

En d'autres termes, tout individu adhérant à l'idéologie djihadiste peut contribuer à retrouver la fierté arabe plus en inspirant la peur qu'en tuant les infidèles et les mauvais musulmans.

Afin d'analyser la manière dont le discours djihadiste diffusé sur internet exploite l'expression des émotions, nous avons examiné les revues djihadistes Dabiq et Dar al-Islam. Dabiq, rédigé en anglais et diffusé depuis le 4 juillet 2014, compte 15 numéros. Dar al-Islam, qui est rédigé en français mais qui n'est pas une traduction de la version anglophone, compte dix numéros, dont le premier a été publié le 23 décembre 2014. Ces deux revues s'adressent à un lectorat qui a déjà adhéré à l'idéologie djihadiste. Bien qu'elle soit diffusée sur internet, la revue présente toutes les caractéristiques d'un magazine en format papier. On y retrouve donc une page de garde, un sommaire, des interviews ainsi que des reportages photos. L'éditeur, qui ne peut pas interagir avec son lectorat, ne peut pas moduler son discours selon son destinataire. Par conséquent, il doit considérer le profil des différents lecteurs qui peuvent accéder à la revue.

Malgré l'importance de l'expression des émotions dans un discours propagandiste, il a été constaté que les réactions émotionnelles sont exprimées directement presque uniquement dans les interviews, où l'énonciateur raconte son expérience pour rejoindre la Syrie, ses combats au front, ou ses journées passées dans le territoire occupé par Daesh. Dans les autres articles, l'éditeur adopte une stratégie différente : il recourt à des scénarios susceptibles d'évoquer une certaine réaction émotionnelle chez le lecteur. Discours et images s'articulent dans une mise en scène des émotions visant à renforcer l'adhésion à l'idéologie djihadiste. Les images des parades ainsi que la description des opérations menées par Daesh ont pour objectif d'exalter le groupe djihadiste et, par conséquent, de nourrir l'amour envers cette communauté. Au contraire, les photos qui montrent les corps égorgés des ennemis et les articles qui condamnent le style de vie occidental visent à alimenter la haine contre l'ennemi de Daesh.

\subsection{Susciter une émotion pour rendre insensibles}

Les revues officielles de Daesh telles que Dar al-Islam et la version anglophone Dabiq ne sont pas le seul moyen adopté pour diffuser l'idéologie djihadiste sur 
internet. Les réseaux sociaux jouent également un rôle important. Si Dar al-Islam et Dabiq s'adressent à un public qui a déjà adhéré à l'idéologie promue par Daesh, les réseaux sociaux constituent souvent la porte d'entrée vers le processus de radicalisation djihadiste (Ducol, 2015). Par conséquent, l'expression des émotions vise ici à amener l'interlocuteur à adhérer à l'idéologie djihadiste. La vidéo Ils te disent, qui fait partie de la campagne lancée par le gouvernement Manuel Valls contre la radicalisation djihadiste, montre la manipulation qui peut avoir lieu sur internet. Dans la vidéo, après avoir regardé des contenus djihadistes sur Facebook, le protagoniste reçoit un message :

(2)

Salut

Cool les trucs que tu like, ça t'intéresse ce ki se passe au Cham en ce moment ?

si ta des questions hésite pas, la vérité elle est là bas, c'est maintenant qu'il faut partir !si tu me donnes ton num j'ai des amis la bas ki se battent jte met en contact.

Bien que ce message ait été produit par le gouvernement, il montre le ton engageant des messages qui peuvent être envoyés par les recruteurs de Daesh. Pouvant interagir directement avec sa cible, le recruteur peut moduler son discours selon son interlocuteur. Comme il a déjà été affirmé, le décalage spatio-temporel des communications médiées par les réseaux permet à l'utilisateur, et dans ce cas au recruteur, de créer une identité $a d$ hoc. L'interlocuteur aura tendance à la percevoir comme vraie car la perception que l'on a d'un individu sur internet est construite à partir des informations que cet individu nous fournit (Mantovani, 2002). Dans le cas des membres ou des sympathisants de Daesh, le fait de choisir une photo de lion comme image du profil sur Facebook a pour objectif de les faire apparaitre comme des individus courageux et valeureux. Cette image de soi finira par être perçue comme vraie par les autres utilisateurs car dans le cyberespace, les internautes tendent à se détacher du monde réel et à percevoir comme réel ce qui se passe dans le monde virtuel. Ce phénomène est analysé en profondeur par Ben-Ze'ev (2005), qui le définit comme detached attachment (" attachement détaché ») ou detattachment (« détattachement »). Plus particulièrement, dans une conversation médiée par les réseaux, l'utilisateur peut ressentir un sentiment d'attraction pour son interlocuteur jusqu'à établir une sorte de relation avec celui-ci. Le recruteur djihadiste vise à susciter des émotions chez son interlocuteur afin d'intensifier ce sentiment d'attraction pour la communauté djihadiste ou, dans certains cas, pour l'un de ses membres. De même, il a pour objectif de faire en sorte que ce detattachment coupe tout lien affectif et émotionnel entre l'interlocuteur et son entourage. En ce qui concerne cette manipulation qui vise à ce que l'individu ne ressente plus d'émotions, le recruteur expose son interlocuteur à des contenus de plus en plus violents afin que sa cible s'habitue à la violence et qu'il n'ait plus peur de mourir. 


\section{Articulation paradoxale des émotions dans la propagande et l'action djihadistes}

Comme nous l'avons vu dans les deux sections précédentes, le lien entre le terrorisme et la peur est évident. Cependant, le discours de Daesh ne se construit pas uniquement autour de la peur et des autres émotions à valence négative. Nous verrons ici comment émotions positives et émotions négatives interagissent dans le discours et l'idéologie djihadistes.

\subsection{Amour et haine dans le discours djihadiste}

Le discours djihadiste ne vise pas uniquement à terroriser l'ennemi. L'objectif de Daesh est également celui de fasciner et d'attirer un nombre important de sympathisants. Pour atteindre cet objectif, Daesh exploite aussi l'expression d'émotions et de sentiments ${ }^{4}$ positifs. Réactions émotionnelles positives et négatives s'articulent donc au sein d'un même discours. Dans la propagande djihadiste, deux sentiments opposés s'alimentent l'un l'autre. Si, d'un côté, la haine contre l'ennemi mécréant renforce l'amour pour la communauté djihadiste, d'un autre côté, l'amour pour la communauté djihadiste alimente le sentiment de haine contre son ennemi.

(3)

Vas-tu laisser le mécréant dormir tranquille dans sa maison pendant que les femmes des musulmans et leurs enfants tremblent de peur, effrayés par le bruit des avions croisés au-dessus de leur tête nuit et jour. Comment peux-tu supporter la vie et arriver à dormir ? Ne vas-tu pas secourir tes frères en jetant la crainte dans le cœur des adorateurs de la croix ? (Dar al-Islam, 2, p. 6$)^{5}$.

Cet exemple, extrait du deuxième numéro de Dar al-Islam, montre comment la haine et l'amour interagissent dans un même discours. Ici, l'énonciateur s'adresse directement au lecteur, en lui posant une question qui a pour objectif de l'inciter à agir contre l'ennemi de Daesh. Plus particulièrement, l'énonciateur souligne le fait que la communauté musulmane qui se trouve dans les zones de conflit au Moyen-Orient subit des frappes aériennes de la part des avions croisés. Cet extrait laisse transparaître la tentative de la part de l'énonciateur de présenter l'Occident comme l'ennemi qui attaque les femmes et les enfants musulmans et, par conséquent, de renforcer la haine que le lecteur ressent envers les occidentaux. De même, en présentant la communauté musulmane comme victime des croisés,

4 Contrairement aux émotions, qui ne durent que quelques secondes, les sentiments ont une durée plus importante. Ekman (1992) identifie six émotions primaires : joie, colère, surprise, tristesse, peur et dégoût.

5 Cet exemple a été extrait du deuxième numéro de la revue de propagande djihadiste francophone Dar al-Islam. Ce numéro a été diffusé en ligne par Al-Hayat, le centre médiatique de Daesh, le 15 février 2015 (consulté le 15 juin 2019 sur le site web https://jihadology.net/). 
l'énonciateur vise à ce que le lecteur soit poussé à aider cette communauté en jetant la crainte dans le cour des adorateurs de la croix. En d'autres termes, dans cet exemple, l'amour pour la communauté est à l'origine de l'action violente contre l'ennemi.

Cette articulation d'émotions positives et négatives peut se présenter aussi en relation à un événement précis. Toutes les attaques terroristes qui ont été menées par Daesh dans ces dernières années ont suscité chez les djihadistes de la joie, de la fierté et d'autres réactions positives. Paradoxalement, il a été possible de constater que ces mêmes attentats ont suscité des réactions positives aussi au sein de la communauté ciblée par Daesh. Suite aux attentats menés en France, par exemple, des manifestations de solidarité et d'amour ont émergé. Quelques jours après les attentats de janvier 2015 contre la rédaction de Charlie Hebdo, les Français ont marché ensemble dans les rues de Paris. De même, suite aux attentats du 13 novembre 2015 à Paris et du 14 juillet 2016 à Nice, le quotidien Le Monde a publié un mémoriel des 159 victimes $^{6}$. Ces portraits nécrologiques ne présentent aucune expression de haine ni de violence. Au contraire, ils sont imprégnés d'émotions et de sentiments positifs comme la joie et l'amour.

\subsection{Une approche d'analyse des émotions dans le discours djihadiste}

Cette analyse a révélé des éléments qui nécessitent d'être considérés lorsque l'on analyse l'expression des émotions dans le discours djihadiste diffusé dans le cyberespace. Le discours examiné doit être analysé dans son contexte de production. Il est donc important de tenir compte du moyen de communication employé et de l'impact que ce dernier a sur le discours diffusé. Nous avons vu que le décalage spatio-temporel des réseaux sociaux influence considérablement les interactions ainsi que la manière d'exprimer une émotion. De même, il est nécessaire de considérer les événements auxquels on fait référence dans le discours ainsi que le point de vue de l'énonciateur. Ce dernier élément est particulièrement important, car il permet d'affirmer si un énoncé exprimant une émotion positive est vraiment un message positif ou si, au contraire, l'objet de telle émotion peut représenter un danger pour la communauté. Comme il a déjà été montré, les attentats perpétrés en France ont suscité chez les djihadistes des émotions positives comme la joie. Cependant, un message exprimant cette joie est un message positif uniquement au niveau de la forme. Le fait que le stimulus de cette émotion positive est un événement négatif révèle la dangerosité potentielle de l'énonciateur.

En outre, comme Shaver, Schwartz, Kirson, et O'Connor (1987) l'affirment, le discours est imprégné des impressions de l'énonciateur même lorsque ce dernier n'exprime pas directement ses émotions. Afin d'examiner le discours djihadiste

6 Le mémoriel est accessible via le lien https://www.lemonde.fr/attaques-aparis/visuel/2015/ 11/25/enmemoire_4817200_4809495.html (page consultée le 15 juin 2019). 
diffusé sur internet à travers l'expression des émotions, il ne faut donc pas se contenter d'étudier uniquement les émotions négatives. Il est également nécessaire de considérer et d'analyser non seulement l'expression des émotions, mais aussi la manière dont le discours est formulé pour susciter des réactions émotionnelles chez l'interlocuteur.

\section{Conclusion}

À travers cette contribution, nous avons proposé une analyse de l'expression des émotions dans un discours propagandiste diffusé sur internet. Cette étude a révélé comment le contexte de production et, plus particulièrement, le moyen de communication, influencent la façon dont les émotions sont exprimées. Nous avons vu comment, lors d'une interaction Facebook, l'énonciateur tend à exprimer directement ses émotions afin de susciter une réaction émotionnelle chez son interlocuteur. Au contraire, ne pouvant pas interagir avec son lectorat, l'auteur d'une revue devra recourir à des scénarios susceptibles de susciter une certaine émotion. Dans le cas du discours djihadiste, l'expression et l'élicitation des émotions ont pour objectif de moduler le comportement de son destinataire. Cette analyse a montré aussi comment l'amour et la haine s'articulent et s'alimentent au sein d'un même discours. Ce résultat nous a permis de définir les éléments à considérer lorsque l'on étudie le discours djihadiste à travers l'expression des émotions.

Autrement dit, cette étude qualitative du discours djihadiste a permis d'aller au-delà des résultats issus d'une analyse quantitative réalisée avec les logiciels Tropes et Iramuteq (Ascone, 2018). Ne pouvant pas tenir compte du point de vue de l'énonciateur, ces outils d'analyse textuelle ne permettent pas d'interpréter les nuances et les visées du discours. Il a ainsi été possible d'identifier les stratégies à visée pathémique qui sont adoptées par Daesh dans les revues Dabiq et Dar al-Islam. Cette approche s'est révélée cruciale dans l'analyse de l'expression des émotions dans le discours djihadiste. Toutefois, une étude d'un corpus construit à partir de réseaux sociaux permettrait de vérifier si les stratégies à visée pathémique adoptées par Daesh varient selon le contexte de production.

\section{References}

Arndt, H., \& Janney R. W. (1991). Verbal, prosodic, and kinesic emotive contrasts in speech. Journal of pragmatics, 15(6), 521-549.

Ascone, L. (2018). La radicalisation à travers l'expression des émotions sur internet (Doctoral dissertation, Université de Cergy-Pontoise).

Ben-Ze'ev, A. (2005). 'Detattachment': the unique nature ofonline romantic relationships. In Y. Amichai-Hamburger (Ed.), The social net: Human behavior incyberspace (pp. 115-138). New York: Oxford University Press.

Caffi C., \& Janney R. W. (1994). Toward a Pragmatics of Emotive Communication. Journal of Pragmatics, 22(3), 325-373.

Christophe, V. (1998). Les émotions : tour d'horizon des principales théories: Vol. 3. Presses Universitaires du Septentrion. DOI: 10.4000/books.septentrion.50970. 
Di Cesare, D. (2017): Terrore e modernità. Torino: Giulio Einaudi Editore.

Ducol, B. (2015). Devenir jihadiste à l'ère numérique. Une approche processuelle et situationnelle de l'engagement jihadiste au regard $d u$ Web (Doctoral dissertation thesis, Université de Laval, Québec, Canada). Retrieved November, 2, 2019, from https://corpus.ulaval.ca/jspui/ bitstream/20.500.11794/25744/1/31398.pdf.

Ekman, P. (1992). An argument for basic emotions. Cognition \& Emotion, 6(3-4), 169-200.

Goossens, V. (2005). Les noms de sentiment. Esquisse de typologie sémantique fondée sur les collocations verbales. Lidil. Revue de linguistique et de didactique des langues, 32, 103-121.

Harkins J., \& Wierzbicka A. (2001). Emotions in crosslinguistic perspective: Vol. 17. Berlin, New York: Walter de Gruyter.

Kramer, A. D., Guillory J. E., \& Hancock J. T. (2014). Experimental evidence of massive-scale emotional contagion through social networks. PNAS. Proceedings of the National Academy of Sciences. DOI: 10.1073/pnas.132004111.

Kramsch, C. J. (2009). The Multilingual Subject: What Foreign Language Learners Say About their Experience and why it Matters. New York: Oxford University Press.

Mantovani, G. (2002). Internet haze: why new artifacts canenhance situation ambiguity. Culture and Psychology 8, 307-326.

Marsella S. C., \& Gratch J. (2009). EMA: A process Model of Appraisal Dynamics. Cognitive Systems Research, 10(1), 70-90.

Plantin, C. (2011). Les Bonnes raisons des émotions : principes et méthode pour l'étude du discours émotionné. Berne: Peter Lang Verlag.

Sapir, E. (1921). Language: an Introduction to the Study of Speech. New York: Harcourt Brace.

Sartre, J. P. (1962). Sketch for a Theory of the Emotions. Hove: Psychology Press.

Scherer, K. R. (2001). Appraisal considered as a process of multilevel sequential checking. In K. R. Scherer, A. Schorr, \& T. Johnstone (Eds.), Appraisal processes in emotion: Theory, methods, research (92-120). New York: Oxford University Press.

Scherer, K. R. (2005). What are Emotions? And how can they be Measured? Social Science Information, 44(4), 695-729.

Shaver, P., Schwartz, J., Kirson, D., \& O'Connor, C. (1987). Emotion knowledge: further exploration of a prototype approach. Journal of personality and social psychology, 52(6). DOI: $10.1037 / / 0022-3514.52 .6 .1061$. 